\title{
A Variable Stiffness Analysis Model for Large Complex Thin-Walled Guide Rail
}

\author{
Xiaolong Wang, Aimin Wang and Yanpeng Lv \\ Beijing Institute of Technology, Department of Mechanical Engineering, 5 South Zhongguancun Street, Haidian District, Beijing, P.R.China
}

\begin{abstract}
Large complex thin-walled guide rail has complicated structure and no uniform low rigidity. The traditional cutting simulations are time consuming due to huge computation especially in large workpiece. To solve these problems, a more efficient variable stiffness analysis model has been propose, which can obtain quantitative stiffness value of the machining surface. Applying simulate cutting force in sampling points using finite element analysis software ABAQUS, the single direction variable stiffness rule can be obtained. The variable stiffness matrix has been propose by analyzing multi-directions coupling variable stiffness rule. Combining with the three direction cutting force value, the reasonability of existing processing parameters can be verified and the optimized cutting parameters can be designed.
\end{abstract}

\section{Introduction}

Large complex thin-walled guide rail has large longitudinal dimension and small cross section which is a typical thin-walled workpiece with low rigidity and high precision requirement. Different processing parameters must be carefully considered in different machining surfaces. To solve these problems, a lot of researches are focused on the cutting simulations [1]-[4]. The optimized cutting parameters can be obtained from simulations. However, this method is not suit for large workpiece due to huge computation. As for large complex thin-walled structure and weak local stiffness, the influence on the deformation of low rigidity caused by cutting force was studied by Sveten et al. [5]. The optimization method of the blade clamping by finite element stiffness analysis simulation was also established [6], [7]. Nevertheless, these studies are all qualitative analysis of the stiffness of the thin-walled workpiece. The quantitative analysis method of the stiffness is rarely mentioned.

The aim of this study is to model the variable stiffness of large complex thin-walled guide rail. Applying one direction's cutting force on sampling points separately and analyzing the deformation on three directions, the value of stiffness can be obtained, and the variable stiffness curves can be drawn. Then, the coupling of three directions' cutting force are studied and the linear superposition relationship is proposed. The calculation method of variable stiffness matrix is given. Combined with the cutting force, the reasonability of existing processing parameters are verified, and the optimized cutting parameters are designed.

\section{Guide rail structure and the clamping form}

The guide rail structure is shown in Fig. 1. The material is 2A12 aluminum alloy with pre-stretching. The cross section is shown in Fig. 2. The size in longitudinal direction is $4 \mathrm{~m}$ and the cross section size is $127^{*} 290$. The guide rail is a typical thin-walled workpiece with complex cross section and low rigidity. The large end face's thickness is only $6 \mathrm{~mm}$. To reduce the machining deformation, the traditional processing usually adopt slow feed, small cutting depth, which can reduce the force in the process of machining. Although this method can reduce deformation, the selection of cutting parameters depends on experience, usually very conservative. So the machining efficiency is very low.

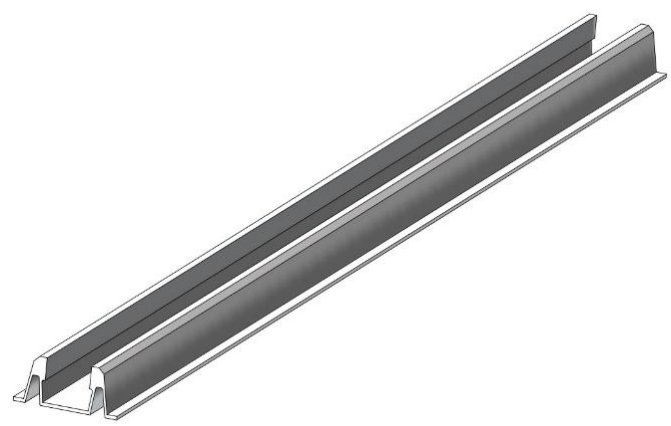

Figure 1. Guide rail structure. 


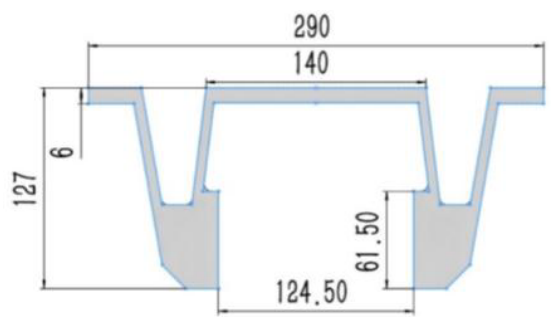

Figure 2. Guide rail cross section.

The machined surface stiffness distribution is different according to the guide rail structure. For example, the inner machined surface's stiffness is higher than the big end surface. So we can optimize its processing efficiency by analyzing its stiffness. The stiffness distribution in inner machined surface is also different among different regions. Further analysis shows even in one exact region, the three directions stiffness are also different. So it's very necessary to obtain the rule of variable stiffness and the quantitative stiffness value to optimize the cutting parameters.

Different clamping form will contribute different stiffness distribution. Fig. 3 shows the clamping form of processing inner machined surface. The big end surface is put on the processing platform, and two wings are clamped by five platens along the longitudinal direction.

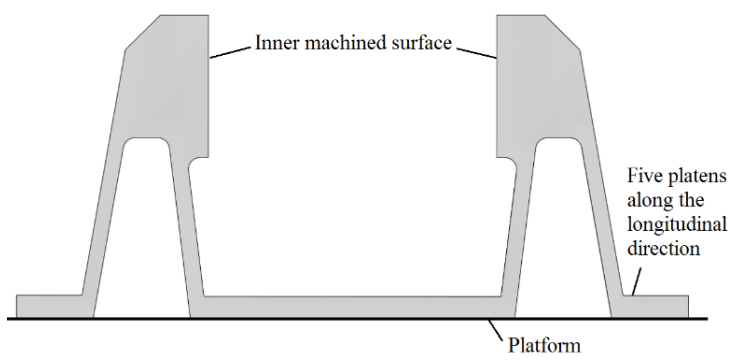

Figure 3. Clamping form of processing the inner machined surface.

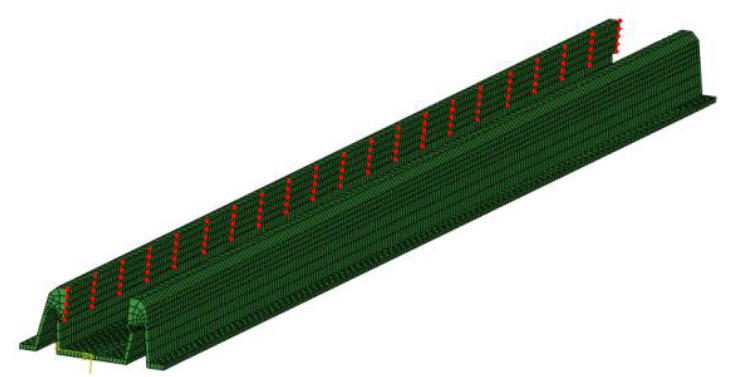

Figure 4. Distribution of sampling points.

\section{Single direction variable stiffness analysis}

\subsection{Distribution of sampling points}

Since the inner machined surface structure is symmetrical, here we just analyze one side. There are 5 evenly distributed sampling points along the cross section direction. The top sampling point's mark x equals 1 . Also there are 21 evenly distributed sampling points along longitudinal direction. The left sampling point's mark y equals 1 . There are a total of 105 samples, and each one has its unique mark x.y, which are shown in Fig. 4.

\subsection{Variable stiffness definition}

The stiffness value is variable in the inner machined surface. The three directions' stiffness of one sampling point is also different. Here we define the y direction stiffness value of a sampling point for single $\mathrm{x}$ direction cutting force $R_{x y}$ is calculated as in Eq. 1 .

$$
R_{x y}=F_{x} / U_{y} \quad(k N / m m)
$$

where $F_{x}$ is the cutting force in $\mathrm{x}$ direction.

So we can describe the single $\mathrm{x}$ direction stiffness of a sampling point by the $R_{x x}, R_{y x}, R_{z x}$ three values.

\subsection{Single direction variable stiffness curve}

Three direction simulated cutting force are applied respectively in a sampling point, and the three direction deformation of a signal direction cutting force can be obtained. According to the definition of variable stiffness, the three stiffness values are calculated. We call that the variable stiffness value curve in one direction is the single direction variable stiffness curve. It can be drawn along the longitudinal direction or cross section direction. For example, we apply the y direction simulated cutting force, with $1 \mathrm{kN}$, in the sampling line 'a' which is consist by all sampling points $\mathrm{x}=\mathrm{a}$ (points $\mathrm{a}{ }^{*}$ ), and the deformation in $\mathrm{y}$ direction can be obtained from ABAQUS. Then, the pints stiffness $R_{y y}$ are calculated and the five variable stiffness curves along longitudinal direction are drawn as Fig. 5.

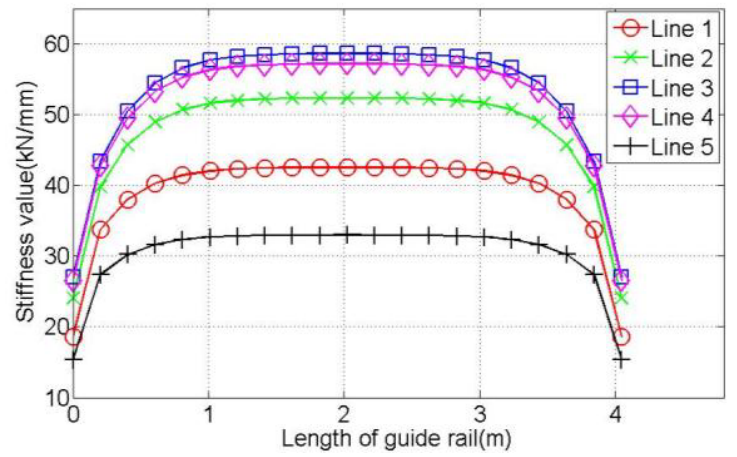

Figure 5. Five variable stiffness curves along longitudinal direction.

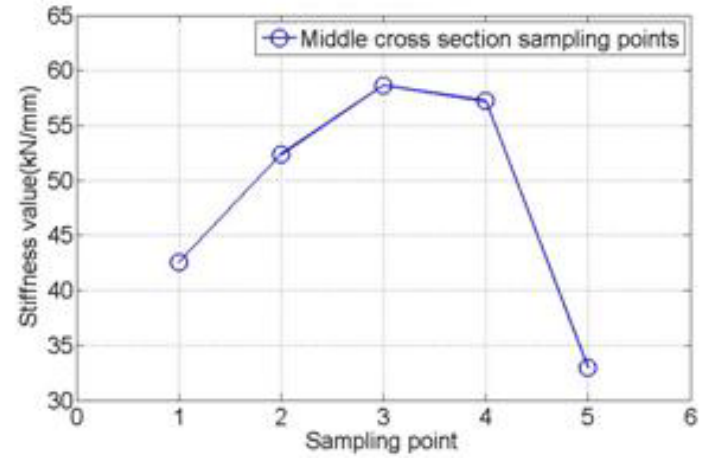

Figure 6. Variable stiffness curve along cross section. 
The variable stiffness curve of middle cross section sampling points is plotted in Fig. 6. This curve is made up of the sampling points $\mathrm{y}=11$ (points *.11).

The simulation results show that the stiffness was significantly lower than the middle stiffness at the ends of the guide rail in the longitudinal direction. The lowest stiffness value was just about $46 \%$ of the highest; In the cross section direction, the stiffness value increased form sampling point 1 to 3 , then decreased until sampling point 5 . The lowest stiffness value was just about $56 \%$ of the highest. Using this method, the same results can be obtained according to $\mathrm{x}$ and $\mathrm{y}$ direction stiffness value.

Thus, the stiffness value on the inner machined surface range is very large, which means higher cutting speed can be used and will not cause large deformation. So the cutting efficiency can be improved.

\section{Multi-directions coupling variable stiffness analysis}

In the process of actual cutting, the cutting force is resultant force of three perpendicular force. The single direction variable stiffness analysis shows that each direction cutting force can cause three directions deformation. So it's necessary to analyze the coupling relationship of variable stiffness. Thus, the variable stiffness model can be used as the guidance in the actual cutting processing.

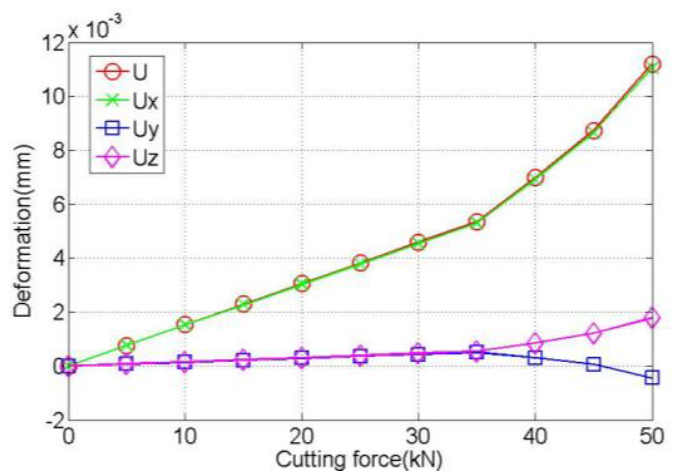

Figure 7. Deformation curves caused by $\mathrm{x}$ direction cutting force.

\subsection{Variable stiffness linear superposition relationship}

The $\mathrm{x}$ and $\mathrm{z}$ multi-directions coupling relationship is studied firstly. Here we take the sampling point 1.1 for example. First obtain the deformation caused by $\mathrm{x}$ direction cutting force using ABAQUS. The cutting force is range from 0 to $50 \mathrm{kN}$. The deformation curves caused by $\mathrm{x}$ direction cutting force are shown in Fig. 7. Where Ux, Uy, Uz are the three directions' deformation, $\mathrm{U}$ is the equivalent deformation. Here we use $f_{x}(F)$ to represent the deformation in this case. Using the same method we can obtain the deformation caused by $\mathrm{z}$ direction force $f_{z}(F)$ which is shown in Fig. 8, and the deformation caused by $\mathrm{x}$ and $\mathrm{z}$ direction force $f_{x z}(F)$ which is shown in Fig. 9. The linear coupling deformation difference value $\Delta f(F)$ curve is shown in Fig. 10, $\Delta f(F)$ is calculated by Eq. 2.

$$
\Delta f(F)=f_{x}(F)+f_{z}(F)-f_{x z}(\mathrm{~F})
$$

The curves from Fig. 7 to Fig. 10 all consist of two part, the linear part and nonlinear part. The nonlinear part is caused by the plastic deformation. Because in this part, the simulating cutting force is too big that the workpiece is yielded. The linear part indicates that the stiffness value is a constant before yield.

The curves in Fig. 7 and Fig. 8 indicate that the cutting force in different direction will cause different deformation in the same direction due to the different stiffness value.

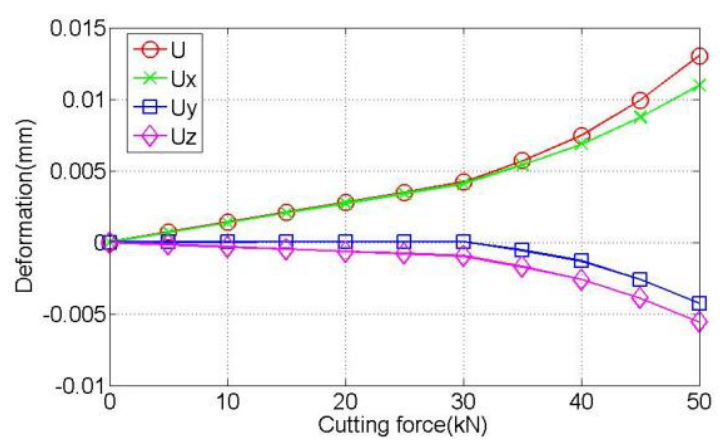

Figure 8. Deformation curves caused by $\mathrm{x}$ direction cutting force.

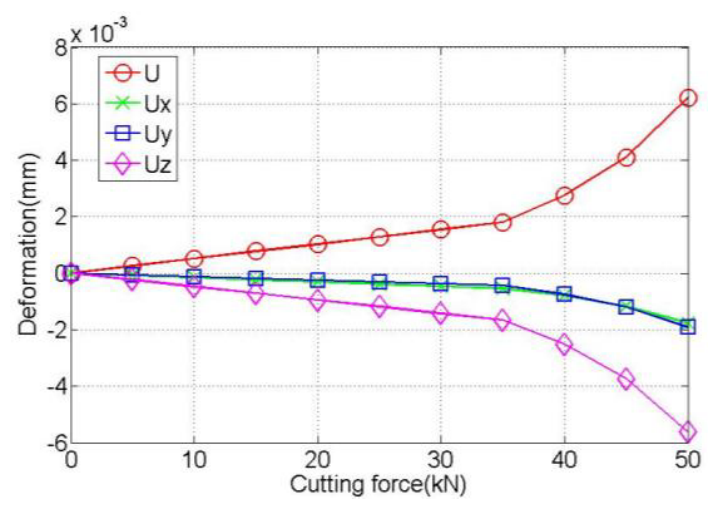

Figure 9. Deformation curves caused by $\mathrm{x}$ and $\mathrm{z}$ direction cutting force.

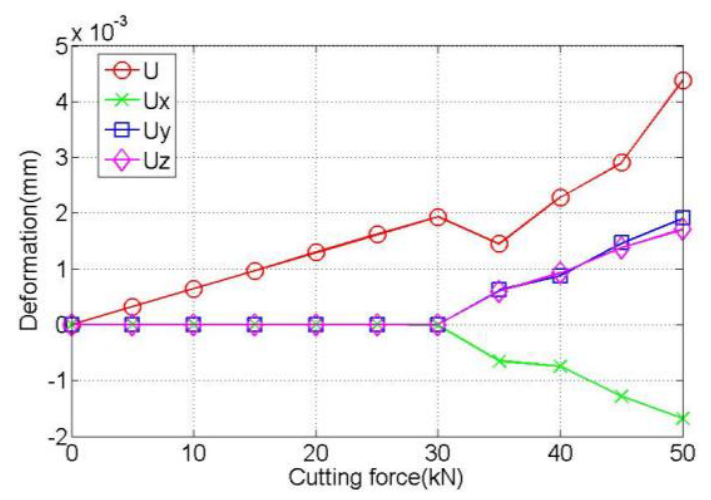

Figure 10. Linear coupling deformation difference value curves.

Fig. 10 shows two kind of coupling relationship. As for the deformation in one direction, the deformation difference value equals to 0 which means the deformation observe linear superposition relationship in elastic stage. However, the equivalent deformation doesn't observe this rule. 
Further study shows that the extensional coupling relationship in three directions is also satisfied linear superposition relationship. Thus this relationship offers a simple method to studied the complex cutting processing by decomposing it into three direction and analyzing respectively. Then we can combine them by linear superposition to guide the actual cutting processing.

It should be noted that, the plastic deformation is only happened in the cutting region in actual cutting processing. Most of the workpiece is still in a low stress elastic state. The influence of low stiffness is reflected in the elastic region. So it's reasonable that we just consider the linear part of the curve.

\subsection{Variable stiffness matrix analysis model}

Assuming that the actual cutting force $F$ is decomposed into three directions as in Eq. 3.

$$
F=\left[\begin{array}{lll}
F_{x} & F_{y} & F_{z}
\end{array}\right]^{T}
$$

Since each direction's cutting force has different influence in the deformation of three directions, there are 9 independent variables in a sampling point. We describe that $R$ is the variable stiffness matrix in Eq. 4. Where the 9 variables represent the stiffness value. The variable stiffness matrix can be obtained from the static analysis simulation by ABAQUS.

$$
R=\left[\begin{array}{lll}
R_{x x} & R_{y x} & R_{z x} \\
R_{x y} & R_{y y} & R_{z y} \\
R_{x z} & R_{y z} & R_{z z}
\end{array}\right]
$$

Assuming that the deformation in three directions is $u$, as in Eq. 5.

$$
u=\left[\begin{array}{lll}
u_{x} & u_{y} & u_{z}
\end{array}\right]^{T}
$$

From the linear superposition relationship, the expression between cutting force, variable stiffness matrix and deformation is shown in Eq. 6.

$$
\left[\begin{array}{l}
u_{x} \\
u_{y} \\
u_{z}
\end{array}\right]=\left[\begin{array}{ccc}
1 / R_{x x} & 1 / R_{y x} & 1 / R_{z x} \\
1 / R_{x y} & 1 / R_{y y} & 1 / R_{z y} \\
1 / R_{x z} & 1 / R_{y z} & 1 / R_{z z}
\end{array}\right]\left[\begin{array}{c}
F_{x} \\
F_{y} \\
F_{z}
\end{array}\right]
$$

The cutting force in three directions can be obtained by simulation or experiment. The cutting parameters are reasonable if the calculated deformation is less than cutting precision. On the other hand, if the cutting precision is given, the allowable maximum cutting force can be obtained.

\section{Conclusion}

Taking the large complex thin-walled guide rail as analysis object, expound the modeling process of the variable stiffness model which can be used to guide the actual cutting processing.

As for a certain clamping structure, the variable stiffness curves are the keys to evaluate the longitudinal and cross section stiffness. According to the coupling cutting force of different direction, the linear superposition relationship is used to couple the stiffness by variable stiffness matrix. The variable stiffness matrix can be obtained from the static analysis simulation by ABAQUS. The time consumption is acceptable even to analyze the large complex thin-walled guide rail.

Compared the simulating deformation which is calculated by cutting force and variable stiffness matrix with cutting precision, the reasonability can be verified, the allowable maximum cutting force can also be obtained. The cutting experiments using the variable stiffness model will be implemented in future work.

\section{References}

1. Yang Y, Wang YL, Li CH. Study on machining distortion of titanium alloy aircraft monolithic component by finite element method and experiment. ADV SCI LETT. 4(8):3206-3210 (2011).

2. Shi GQ, Deng XM, Shet C . A finite element study of the effect of friction in orthogonal metal cutting. Finite Elem Anal Des, 38:863-883 (2002).

3. Yong Yang, Weiwei Zhu, Study on cutting temperature during milling of titanium alloy based on FEM and experiment. Int. J. Adv. Manuf. Technol.73(9):1511-1521 (2014).

4. Ratchev S, Liu S, Becker A A. Error compensation strategy in milling flexible thin-wall parts $[\mathrm{J}]$. J. Mater. Process. Technol.162(5):673-681 (2005).

5. R Svetan, W Huang, W Liu. Modeling and simul ation en-vironment for machining of low-rigidity comp onents [J]. J. Mater. Process. Technol. 153:67 - 73 (2004).

6. H. Wang, L. Huang, C. Yao, et al.Integrated Analysis Method of Thin-Walled Turbine Blade Precise Machining[J]. Int. J. Precis. Eng. Manuf. 16(5):1011-1019 (2015).

7. Wang, Y., Chen, X., Gindy, N., et al. Elastic Deformation of a Fixture and Turbine Blades System based on Finite Element Analysis, Int. J. Adv. Manuf. Technol.36(4):296-304 (2008). 